\section{Isolated ileal perforation in infancy: a lethal initial presentation of Hirschsprung's disease}

\author{
Fadi Iskandarani,1,2 \\ Chawki Hammoud,1,2 Sarah Srour, 1,2 \\ Gloria Pelizzo, ${ }^{3}$ Ghassan Nakib, ${ }^{4}$ \\ Valeria Calcaterra,5,6 Amir Khanafer 1,2 \\ 1 Faculty of Medicine, Lebanese \\ University, Hadath, Beirut, Lebanon; \\ 2Sahel General Hospital, Ghobeiri, \\ Beirut, Lebanon; 3Pediatric Surgery \\ Unit, Children's Hospital, Istituto \\ Mediterraneo di Eccellenza Pediatrica, \\ Palermo, Italy; ${ }^{4}$ Department of Pediatric \\ Surgery, Medclinic Middle East, \\ Mediclinic City Hospital Dubai, United \\ Arab Emirates; 5Pediatric Unit, \\ Department of Maternal and Children's \\ Health, Fondazione IRCCS Policlinico \\ San Matteo, Pavia; 'Department of \\ Internal Medicine, University of Pavia, \\ Italy
}

\begin{abstract}
A rare case of ileal perforation, as a fatal initial presentation of total colonic aganglionosis (TCA) in infancy is reported. A 10 -week-old boy, was brought to the emergency department with symptoms of complicated intestinal obstruction. He looked ill, was lethargic, markedly dehydrated and had a severely distended abdomen. An abdominal X-ray revealed multiple air fluid levels seen in a distended small intestine. During exploratory laparotomy the ileum was massively dilated with distal segment perforation. Ileal perforation repair was performed. A totally collapsed microcolon was identified. Biopsies were taken from the high rectum, sigmoid and hepatic flexure. Appendectomy and ileostomy were performed. All biopsies, as well as the appendix, showed absence of ganglion cells. Despite this procedure the patient progressively deteriorated and later died due to sepsis. Ileal perforation in infants is a rare, but potentially fatal initial presentation of TCA. Early detection is essential to prevent life-threatening complications.
\end{abstract}

\section{Introduction}

Hirschsprung's disease (HD) is a relatively common developmental disorder characterized by congenital aganglionosis of Auerbach's myenteric and Meissner's sub- mucosal neural plexuses of the distal colon, of varying length, resulting in a functional obstruction. The overall incidence of sporadic HD is 1 in 5000 live births, ${ }^{1}$ with maleto-female ratio of $3: 1$ to $4: 1,2,3$ but approaching $1: 1$ when the whole colon is involved. Total colonic aganglionosis (TCA) is a severe and uncommon form of HD. It occurs in approximately $2-13 \%$ of all patients with the disease and involves the entire colon, but may extend proximally into varying lengths of small bowel. Late diagnosis of TCA often occurs, due to milder symptoms; this delay however increases the risk of severe complications, mainly perforation, enterocolitis, sepsis and death, reaching 20 to $50 \% .^{1-4}$

Total colonic HD with an isolated ileal perforation in the neonatal period, as the primary presentation, is very rare, particularly in infants. 5,6

We described a case of TCA in a 10 weeks old boy, previously healthy, presenting with abdominal distension due to an intestinal obstruction, complicated by ileal perforation, enterocolitis, sepsis and death. A literature review of this form of HD is also discussed.

\section{Case Report}

A 10 weeks old male infant, with no relevant medical history, was brought to the emergency department with severe abdominal distention and lethargy.

Three days prior to presentation, the mother noted fever, poor feeding and vomiting which was mainly post-prandial, nonprojectile, non-bilious and non-bloody. The infant's condition deteriorated one day prior to presentation, with cessation of bowel movements and subsequent progressive abdominal distention and irritability at first, then lethargy.

The baby was born at term by vaginal delivery, with a weight of 1.860 grams (small for gestational age). No delay in passing meconium at birth nor history of constipation were reported. He was being breast fed and his vaccination schedule was up to date. No family history of Hirschsprung's disease or congenital disorders was present.

On physical examination, the baby measured $30 \mathrm{~cm}$ long $(<3$ percentile for age and sex) and weighed 3430 grams ( $<3$ percentile for age and sex). He was afebrile $\left(37^{\circ} \mathrm{C}\right)$, fair heart rate $(124$ beats/minute), tachypneic (45 breaths/ minute), hypotensive (60-35 $\mathrm{mmHg}$ ), and maintained an oxygen saturation on room air of $97 \%$. He looked unwell, lethargic (low activity, weak crying, poor sucking), markedly dehydrated
Correspondence: Fadi Iskandarani, Sahel General Hospital, PO Box 99/25, Ghobeiri, Beirut, Lebanon.

Tel.: +961.3231.626.

E-mail: iskandaranifadi@gmail.com

Key words: Hirschsprung's disease; total colonic aganglionosis; ileal perforation; initial presentation; infant.

Acknowledgments: the authors thank Dr. Andrew Mccomb for English revision of the manuscript.

Contributions: FI, SS, AK management of the patient, drafting the article, critical revision of the article; GP, VC, GN drafting the article, literature review, critical revision of the article.ù

Conflict of interest: the authors declare no potential conflict of intersest.

Received for publication: 8 February 2017. Revision received: 27 March 2017.

Accepted for publication: 28 March 2017.

This work is licensed under a Creative Commons Attribution NonCommercial 4.0 License (CC BY-NC 4.0).

CCopyright F. Iskandarani et al., 2017

Licensee PAGEPress, Italy

Pediatric Reports 2017; 9:7084

doi:10.4081/pr.2017.7084

(decreased skin turgor, pale mottled skin), and had a markedly distended abdomen which was tense and tender to palpation with reduced bowel sounds (agitation and crying).

Laboratory investigations revealed a high leukocyte count of $20.56 \times 109 / \mathrm{L}$ neutrophilia $(41 \%), \quad$ thrombocytosis $(994 \times 109 / \mathrm{L})$, elevated C-reactive protein (25.5 U/L), electrolyte disturbance (hyponatremia $129 \mathrm{mEq} / \mathrm{L}$ ), hyperglycemia (196 $\mathrm{mg} / \mathrm{dL}$ ), elevated liver function tests (SGOT $164 \mathrm{U} / \mathrm{L}$ and SGPT $232 \mathrm{U} / \mathrm{L}$ ) and compensated metabolic acidosis on arterial blood gas test $\left(\mathrm{PH} 7.324, \mathrm{PaCO}_{2} 34.2\right.$ and $\mathrm{HCO}_{3}$ 17.2). A plain abdominal $\mathrm{X}$-ray revealed multiple air fluid levels seen in distended loops of small intestine; no pneumoperitoneum was noted (Figure 1).

The baby was treated for clinical sepsis, which involved immediate fluid resuscitation and initiation of broad spectrum parenteral antibiotics. An initial diagnosis of complicated intestinal obstruction was made and urgent laparotomy was performed. During surgery, the ileum was massively dilated with identification of a distal segment perforation (10 inches from ileocecal valve). Primary repair of the ileal perfo- 
ration was done. A totally collapsed microcolon was clearly noted which suggested the diagnosis of HD. A transition zone was identified at the ileocecal valve. Full thickness biopsies were taken from the high rectum, the sigmoid colon and the hepatic flexure. Appendectomy and protective ileostomy (ileum proximal to transition zone) were performed. All biopsies, as well as the appendix, showed absence of ganglion cells. Histological evidence of enterocolitis was also noted.The child progressively deteriorated after the procedure despite good resuscitation, intubation and monitoring in the neonatal intensive care unit. Cardio-pulmonary arrest occurred twice after the procedure: at eight hours with successful resuscitation and at twenty hours. Resuscitation was unsuccessful after this second arrest. Autopsy was not performed at the parents' request. The final diagnosis was ileal perforation associated with enterocolitis, sepsis and septic shock, in a case of TCA.

\section{Discussion}

A case of ileal perforation associated with enterocolitis, as a fatal initial presentation of TCA in an infant older than 2 months is reported.

Hirschsprung's disease is a relatively common developmental disorder in neonates characterized by aganglionosis in the distal colon, resulting in a functional obstruction. Clinical classification of HD has evolved with further understanding of the disease, from ultra-short, short and long segment HD, to colonic and TCA with or without involvement of the small bowel.1-4

Total colonic aganglionosis represents a relatively uncommon form of $\mathrm{HD}$ and is defined as aganglionosis extending from the anus to at least the ileocaecal valve but involving no more than $50 \mathrm{~cm}$ of small bowel proximal to the ileocaecal valve. Even thought, in this case, there was no related family history of HD, nor any and associated anomalies found, it had been reported that the familial incidence of TCA is higher $(12.4 \%$ to $33 \%)$ than that in patients with classical HD. A number of developmental conditions, such as chromosomal and congenital hypoventilation syndrome as well as ileal atresia and tumors of neural origin have been also associated with TCA. ${ }^{1-6}$

Compared to the classic cases of HD, TCA presents more diagnostic problems and is usually associated with higher morbidity and mortality rates. ${ }^{1-3}$

The initial presentation of HD is often as a functional obstruction at or shortly after birth (failure to pass meconium within 24$48 \mathrm{~h}$ of birth, abdominal distension, bilious emesis, and enterocolitis). Paradoxically later presentation is not uncommon in TCA, where passage of meconium is often reported. These cases can easily be missed without a high index of suspicion.

Isolated ileal perforation in infants as a primary presentation is uncommon. In the literature, 66 documented cases of HD in which bowel perforation was a primary presenting sign were reported, but only in 4 of these was the ileum involved.7-26 The addition of our case brings this total to five. All these reported cases had TCA; only our infant was older than 2 months at initial presentation (Table 1). ${ }^{7-26}$

Aganglionosis is the most common cause of large bowel obstruction in the young infant and therefore colonic or small bowel perforation should raise the suspicion of HD. As reported by Newman, ${ }^{6}$ where bowel perforation occurred early in the course of HD, the majority of cases (62\%) were associated with long-segment HD or TCA. The most common sites of perforation were the proximal colon $(68 \%)$ and appendix $(18 \%)$ or distal small bowel $(6 \%) .5,6$ In cases with a short or intermediate length of aganglionic bowel, the perforation was proximal to or at the site of transition, but in $84 \%$ of infants with TCA the perforation was situated in aganglionic bowel. The mechanism of perforation in HD appears to be directly related to increased intraluminal pressure from distal obstruction, rather than any inflammatory process, subsequent bacterial overgrowth or intestinal wall ischemia. ${ }^{1,24}$

In this case enterocolitis was associated with perforation in TCA. This association is infrequently reported in infants less than 1 year old, $7-26$ and in the majority of cases, absence of enterocolitis was inferred from the case descriptions.

Theoretically, every HD patient is at risk for developing associated enterocolitis. Studies have shown that several factors contribute to this risk, such as family history, long segment disease and previous episodes of enterocolitis. ${ }^{1-6,27}$ Our little patient had no personal or familial anamnestic data of enterocolitis, but pathology results confirmed the long segment character of the disease. Additionally, as in our report, untreated HD patients are at high risk of associated enterocolitis. Pathologically, enterocolitis is defined as an inflammation of the lining of either the colon or the small intestine. As the disease progresses, the lining erodes, and the inside of the intestine becomes filled with pus. ${ }^{27}$ If the process is left to progress untreated, patients become very ill and perforation of the intestine may occur. Enterocolitis is

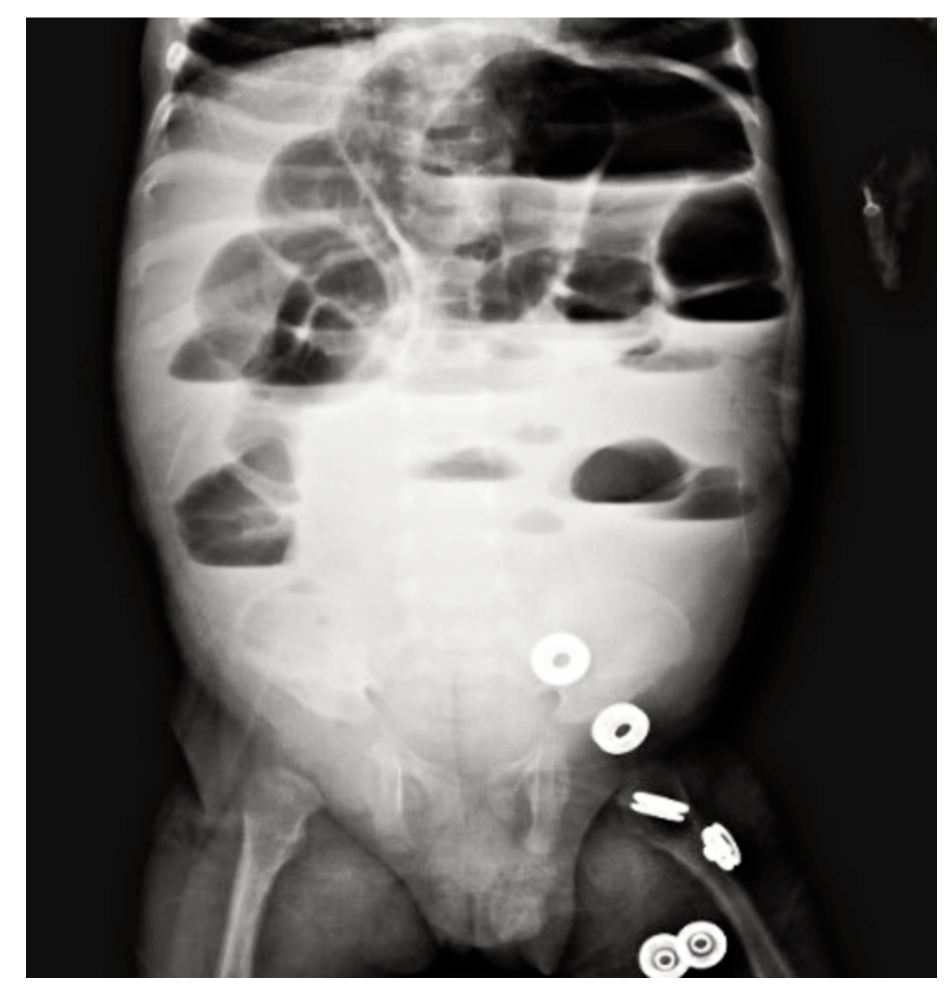

Figure 1. Multiple air fluid levels seen in distended small intestine, but no pneumoperitoneum. 
usually accompanied by abdominal distension in $99 \%$ of cases, explosive diarrhea in $82 \%$, vomiting in $61 \%$, fever in $40 \%$, lethargy in $32 \%$, rectal bleeding in $6 \%$, shock in $6 \% .^{2}$ Our baby presented with acute onset abdominal distension and lethargy. Fever and vomiting were reported by the mother several days earlier, responsive to anti-pyretics which were administered rectally, but no actual documentation of fever exists before and during hospitalization. Enterocolitis in patients with HD may be considered a risk factor for the fulminant, rapidly progressive and lethal form, with subsequent death, especially in the context of a late presentation. ${ }^{1-4,} 8,10$

Total colonic aganglionosis presents other diagnostic problems. X-ray studies may show enlarged loops of bowel, and a contrast enema may show a question markshaped colon owing to the rounded edges of the large intestines. However, there are no specific pathognomonic finding on barium enema and X-ray studies are diagnosticin only $20 \%$ to $30 \%$ of all patients with TCA. ${ }^{3,4}$ The diagnosis of TCA must be entertained if clinical symptoms of intestinal obstruction persist in the absence of any other known causes despite a radiologically normal-looking colon. ${ }^{3}$ The definitive diagnosis of TCA is obtained by performing laparotomy and intraoperative seromuscular biopsies of the rectum, colon, and ileum. In our case, during the laparotomy, the transitional zone was very well identified at the ileocecal valve; the entire colon was collapsed and the distal ileum was very dilated and perforated around 10 inches proximal to the valve. This strongly suggested a diagnosis of TCA, which was confirmed by the biopsy results; because of the absence of an ileal biopsy, the ileal involvement remains unknown.

Ultimate treatment for TCA is surgical. Despite recent advances in surgical techniques and meticulous pre- and post-operative management, surgical treatment for TCA remains challenging. The affected patients often require multiple procedures; longterm results are suboptimal and complications are common. ${ }^{1-4}$ In this report, definitive surgery was not performed as the condition of the baby was critical and his small bowel severely inflamed. A protective ileostomy only was created with definitive surgery planned once there had been improvement of the general status of the infant.

Table 1. Literature survey of patients with Hirschsprung's disease presenting with ileal or other location of perforation as initial presentation

\begin{tabular}{|c|c|c|c|c|}
\hline Reference & patients & Age & Location of perforation & Extent of aganglionic segment \\
\hline \multicolumn{5}{|c|}{ Ileal perforation } \\
\hline Bowden et al. ${ }^{7}$ & 1 & 18 days & Terminal ileum & Total colonic aganglionosis \\
\hline Keefer ${ }^{8}$ & 1 & 13 days & Terminal ileum & Total colonic aganglionosis \\
\hline Patel et al. 5 & 1 & 2 days & Terminal ileum & Total colonic aganglionosis \\
\hline Swenson et al. ${ }^{9}$ & 1 & Newborn & Ileum & Total colonic aganglionosis \\
\hline This report & 1 & 2 months and 2 weeks & Terminal ileum & Total colonic aganglionosis \\
\hline \multicolumn{5}{|c|}{ Other location of perforation } \\
\hline Arliss et al. 10 & 1 & 7 days & Appendix & Rectosigmoid \\
\hline Asch et al. ${ }^{11}$ & 2 & 8 weeks & Appendix & Total colonic aganglionosis \\
\hline Bowden et al. ${ }^{7}$ & 1 & 5 days & Cecum & Total colonic aganglionosis \\
\hline Donald et al. ${ }^{12}$ & 2 & 2 days; 4 days & Cecum & Sigmoid; transverse colon \\
\hline Forshall et al. ${ }^{13}$ & 1 & 4 days & Cecum & Ascending colon \\
\hline Frech $^{14}$ & 4 & 1 month & Colon & Total colonic aganglionosis \\
\hline Gastrin et al. 15 & 1 & 11 days & Appendix & Disatl half colon \\
\hline Grobl6 & 3 & 1-2 days & Cecum (1); splenic flexure (2) & Distal (2); not recorded (1) \\
\hline Hiatt17 & 2 & Newborns & Sigmoid colon & Rectosigmoid \\
\hline Leonidas et al. ${ }^{18}$ & 1 & 2 days & Cecal perforation with enterocolitis & Distal \\
\hline Martin et al. 19 & 3 & 1 day; 15 days; 33 days & Appendix & Transverse colon; splenic flexure (2) \\
\hline Newman et al. ${ }^{6}$ & 3 & 1 day; 2 days; 4 months & Cecum (2); appendix & Distal (sigmoid); total colonic aganglionosis (2) \\
\hline Ojayi et al. ${ }^{20}$ & 2 & 1 day & Cecum & Distal \\
\hline Sane et al. ${ }^{21}$ & 2 & 1 month & Proximal colon (2) & Total colonic aganglionosis \\
\hline Sarioglu et al.22 & 2 & 40 days; 27 days & Appendix & Total colonic aganglionosis; descending colon \\
\hline Sing et al. ${ }^{23}$ & 14 & Newborns & $\begin{array}{l}\text { Transverse-sigmoid colon or splenic flexure } \\
\text { (14, but not individually defined) }\end{array}$ & Not defined \\
\hline Soltero-Harrington et al. 24 & 1 & 6 weeks & Ascending colon & Total colonic aganglionosis \\
\hline Soper et al. ${ }^{25}$ & 1 & 1 day & Cecum & Sigmoid \\
\hline Swenson et al. ${ }^{9}$ & 15 & Newborns & Colon (14); appendix (1) & $\begin{array}{l}\text { Descending colon (2); transverse colon (4); } \\
\text { cecum (1); sigmoid (5); terminal ileum (2); } \\
\text { total colonic aganglionosis (1) }\end{array}$ \\
\hline Wylie 26 & 1 & 7 days & Appendix & Not recorded \\
\hline
\end{tabular}




\section{Conclusions}

Ileal perforation in infants is a rare but potentially lethal initial presentation of TCA. TCA must be a major differential consideration in all neonates or young infants with persistent symptoms referrable to the gastrointestinal tract even when no delay in passing meconium at birth was reported. It is challenging, but imperative to establish early diagnosis and proper management in order to prevent unexpected progression of the disease to life threatening complications.

\section{References}

1. Kenny SE, Tam PK, Garcia-Barcelo M. Hirschsprung's disease. Semin Pediatr Surg 2010;19:194-200.

2. Chen F, Winston JH 3rd, Jain SK, Frankel WL. Hirschsprung's disease in a young adult: report of a case and review of the literature. Ann Diagn Pathol 2006;10:347-51.

3. Anupama B, Zheng S, Xiao X. Ten-year experience in the management of total colonic aganglionosis. J Pediatr Surg 2007;42:1671-6.

4. Moore SW, Total colonic aganglionosis and Hirschsprung's disease: a review. Pediatr Surg Int 2015;31:1-9.

5. Patel RV, Lawther S, Mccallion WA. Discordant monozygotic total colonic Hirschsprung's disease presenting with neonatal isolated ileal perforation. BMJ Case Rep 2013;2013:bcr2013200743..

6. Newman B, Nussbaum A, Kirkpatrick JA, Colodny A. Appendiceal perforation, pneumoperitoneum, and
Hirschsprung's disease. J Pediatr Surg 1988;23:854-6.

7. Bowden DH, AM, Munn JD. Hirschsprung's disease in the neonatal period: a report of five cases, four of which involved the small intestine. J Pediatr 1957;50:321-6.

8. Keefer GP, Mokrohisky JF. Congenital megacolon (Hirschsprung's disease). Radiology 1954;63:157-74.

9. Swenson 0, Sherman JO, Fisher JH. Diagnosis of congenital megacolon: an analysis of 501 patients. J Pediatr Surg 1973;8587-94.

10. Arliss J, Holgersen LO. Neonatal appendiceal perforation and Hirschsprung's disease. J Pediatr Surg 1990;25:694-5.

11. Asch MJ, Weitzmann JJ, Hays DM, et al. Total colon aganglionosis. Arch Surg 1972; 105:74-8.

12. Forshall I, Rickham P, Mossman DB. Functional intestinal obstruction in the newborn. Arch Dis Child 1951;26:2949.

13. Donald JG, Donald JW. Unusual manifestations of aganglionic disorder in the newborn. Surgery 1964;56:644-50.

14. Frech RS. Aganglionosis involving the entire colon and a variable length of small bowel. Radiology 1968;90:24957.

15. Gastrin U, Josephson S. Appendiceal peritonitis and megacolon in the neonatal period. Acta Chir Scand 1970;136:153-5.

16. Grob M. Intestinal obstruction in the newborn infant. Arch Dis Child 1960;35:40-50.

17. Hiatt RB. The pathologic physiology of congenital megacolon. Ann Surg 1951;133:313-20.
18. Leonidas JC, Krasna IH, Strauss L, et al. Roentgen appearance of the excluded colon after colostomy for infantile Hirschsprung's disease. Am J Roentgenol Radium Ther Nucl Med 1971;112:116-22.

19. Martin LW, Perrin EV. Neonatal perforation of the appendix in association with Hirschsprung's disease. Ann Surg 1967;166:799-802 .

20. Ojayi OOA, Solanke TF, Bohrers P, Seriki O. Hirschsprung's disease in the neonate presenting as cecal perforation. Pediatrics 1969;43:102-6.

21. Sane SM, Girdany BA. Total aganglionosis cdi. Radiology 1973;107:394404.

22. Sarioglu A, Tanyel FC, Buyukpamukcu N, Hicsonmez A. Appendiceal perforation: a potentially lethal initial mode of presentation of Hirschsprung's disease. J Pediatr Surg 1997;32:123-4.

23. Singh S, Rawat J, Wakhlu A, et al. Sixyear retrospective analysis of colonic perforation in neonates and infants: single centre experience. Afr J Paediatr Surg 2012;9:102-5.

24. Soltero-Harnngton LA, Garcia-Ainaldi A, Able LW. Total aganglionosis of the colon: recognition and management. J Pediatr Surg 1969;4:330-8.

25. Sopor AT, Opitz JM. Neonatal pneumopentoneum and Hirschsprung's disease. Pediatr Surg 1962;51:527-33.

26. Wylie GG. Course and management of Hirschsprung's disease. Lancet 1957; 272:847-55.

27. Elhalaby EA, Teitelbaum DH, Coran AG, Heidelberger KP. Enterocolitis associated with Hirschsprung's disease: a clinical histopathological correlative study. J Pediatr Surg 1995;30:1023-6. 